\title{
Modelling on vibration of skew plate with thickness and temperature variation
}

\author{
Reeta Bhardwaj ${ }^{1}$, Naveen Mani $^{2}$ \\ ${ }^{1}$ Department of Mathematics, Amity University Haryana, Gurgaon, India \\ ${ }^{2}$ Department of Mathematics, Sandip University, Nashik, Maharshtra, India \\ ${ }^{2}$ Corresponding author \\ E-mail: 1bhardwajreeta84@gmail.com, ${ }^{2}$ naveenmani81@gmail.com \\ Received 14 January 2019; accepted 26 January 2019 \\ DOI https://doi.org/10.21595/vp.2019.20544
}

Check for updates

Copyright $(2019$ Reeta Bhardwaj, et al. This is an open access article distributed under the Creative Commons Attribution License, which permits unrestricted use, distribution, and reproduction in any medium, provided the original work is properly cited.

\begin{abstract}
The present model describes the vibrational analysis of skew (parallelogram) plate made up of non-homogeneous material with variable thickness and temperature on clamped edges. One dimensional thickness and two-dimensional temperature variation is taken into consideration. The non-homogeneity of the plate varies circular in one dimension. The governing differential equation of motion for vibration analysis is solved by using Rayleigh Ritz technique and time period is calculated for the combination of different variation of plate parameters. The obtained results are presented in tabular form.
\end{abstract}

Keywords: vibration, linear variation, circular variation, thickness.

\section{Introduction}

Non-homogeneous plate with variable thickness is widely used in structural applications because of their high strength, light weight, and having higher stiffness. Therefore, in order to make good structural applications, it is required to study vibration characteristics of such plates. Many researchers have been worked in this direction and applied various method to solve vibration problem.

Ritz variational method [1] is applied to solve vibration problems of skew plates on different edge conditions and natural frequencies, modes shape is obtained for side ratio and skew angle. Natural vibration of skew plates [2] is presented on eight edge conditions by using the new version of differential quadrature method (DQM). Vibrational analysis of several laminated composite doubly-curved shells, singly-curved shells and plates with variable thickness is analyzed using generalized differential quadrature (GDQ) method [3]. Differential quadrature method (DQM) is used to study the effects of thickness-to-length ratio, volume fraction index, temperature, geometrical shape, and the boundary conditions on the frequency parameters of the functionally graded (FG) quadrilateral plates [4]. The vibrational characteristics of the functionally graded (FG) quadrilateral microplates [5] are investigated by using modified strain gradient theory (MSGT) on different boundary conditions. Free vibration analysis of functionally graded (FG) rectangular plates [6] on simply supported and clamped edge conditions under temperature field is studied and natural frequencies are obtained by Ritz method. Vibrational analysis of two types of functionally graded material (FGM) sandwich plates [7] with nonlinear temperature variation along with thickness is presented by using finite element (layer wise) formulation. Vibration of skew plates [8] with large skew angle is studied by using moving least square Ritz (MLS-Ritz) method and the results are obtained for rhombic plates on various edge conditions. Free vibration analysis of thin laminated skew plates [9] on clamped edges is investigated by using finite strip transition matrix method. Free vibration of rectangular and skew multilayered plate [10] are presented by using variable-kinematic Ritz method on different boundary conditions and evaluated convergence and accuracy of method. The transverse vibrations of skew plates [11] with variable thickness on different combinations of boundary conditions is presented by using Rayleigh Ritz method. The finite strip method [12] is employed to study the free vibration of skew orthotropic plates, with two opposite edges simply supported and the other two edges are free. 
Vibrational of non-homogeneous skew plate [13] with varying thicknesses and temperature is analyzed by using Rayleigh Ritz technique. Natural frequency of skew plate [14] with variation in thickness and Poisson's ratio is studied by using Rayleigh Ritz method. A model is analyzed to study the vibration of tapered skew plate [15] on clamped edges using Rayleigh Ritz method. Free vibrational analysis of non-homogeneous parallelogram plate [16] with two dimensional circular variations in thickness along with temperature is studied using classical plate theory (CPT). Vibrational analysis of tapered square plate [17] with linear variation in density and bi linear variation in temperature is described using Rayleigh Ritz technique. Effect of plate parameters on time period of rectangular plate is studied in [18] using Rayleigh Ritz technique.

In this study, we computed time period of skew plate on clamped edges under different variation of parameters and results are presented in tabular form.

\section{Analysis}

Consider a thin parallelogram plate made up of non-homogeneous material with skew angle $\theta$ as shown in Fig. 1 referred to skew coordinates $\zeta^{\prime}=\zeta-\psi \tan \theta, \psi^{\prime}=\psi \sec \theta$.

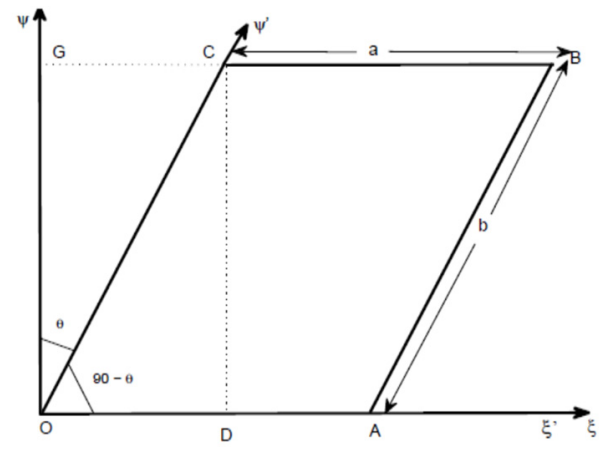

Fig. 1. Skew plate with angle $\theta$

The expression for maximum kinetic energy $T_{s}$ and strain energy $V_{S}$ in skew coordinates is:

$$
\begin{aligned}
& T_{s}=\frac{1}{2} \omega^{2} \cos \theta \iint \rho l \Phi^{2} d \psi^{\prime} d \zeta^{\prime}, \\
& V_{s}=\frac{1}{2 \cos ^{3} \theta} \iint D_{1}\left[\begin{array}{c}
\left(\frac{\partial^{2} \Phi}{\partial \zeta^{\prime 2}}\right)^{2}-4 \sin \theta\left(\frac{\partial^{2} \Phi}{\partial \zeta^{\prime 2}}\right)\left(\frac{\partial^{2} \Phi}{\partial \zeta^{\prime} \partial \psi^{\prime}}\right) \\
+2\left(\sin ^{2} \theta+v \cos ^{2} \theta\right)\left(\frac{\partial^{2} \Phi}{\partial \zeta^{\prime 2}}\right)\left(\frac{\partial^{2} \Phi}{\partial \psi^{\prime 2}}\right) \\
+2\left(1+\sin ^{2} \theta-v \cos ^{2} \theta\right)\left(\frac{\partial^{2} \Phi}{\partial \zeta^{\prime} \partial \psi^{\prime}}\right)^{2} \\
-4 \sin \theta\left(\frac{\partial^{2} \Phi}{\partial \zeta^{\prime} \partial \psi^{\prime}}\right)\left(\frac{\partial^{2} \Phi}{\partial \psi^{\prime 2}}\right)+\left(\frac{\partial^{2} \Phi}{\partial \psi^{\prime 2}}\right)^{2}
\end{array}\right] d \psi^{\prime} d \zeta^{\prime},
\end{aligned}
$$

where, $D_{1}=E l^{3} / 12(1-v)$ is flexural rigidity, here $E, l$ and $v$ are Young's modulus, thickness and Poisson's ratio of the plate.

\section{Construction of problem}

Consider a parallelogram plate of length $a$ and breadth $b$ with linear variation in thickness and circular variation in density and Poisson's ratio as: 


$$
\begin{aligned}
& l=l_{0}\left(1+\beta \frac{\zeta^{\prime}}{a}\right), \rho=\rho_{0}\left[1-m_{1}\left(1-\sqrt{1-\frac{\zeta^{\prime 2}}{a^{2}}}\right)\right], \\
& v=v_{0}\left[1-m_{2}\left(1-\sqrt{1-\frac{\zeta^{\prime 2}}{a^{2}}}\right)\right],
\end{aligned}
$$

where $\beta,(0 \leq \beta \leq 1)$ is tapering parameter of the plate and $m_{1}, m_{2}\left(0 \leq m_{1}, m_{2}<1\right)$ is non-homogeneity parameters.

The two-dimensional steady temperature variation is considered as:

$\tau=\tau_{0}\left(1-\frac{\zeta^{\prime}}{a}\right)\left(1-\frac{\psi^{\prime}}{b}\right)$

where $\tau$ and $\tau_{0}$ are the temperature on the plate at any point and at the origin respectively. The temperature dependent modulus of elasticity is:

$E=E_{0}(1-\gamma \tau)$,

where $E_{0}$ is the Young's modulus at $\tau=0$ and $\gamma$ is slope of variation.

Substituting Eq. (4) in Eq. (5) we get:

$E=E_{0}\left[1-\alpha\left(1-\frac{\zeta^{\prime}}{a}\right)\left(1-\frac{\psi^{\prime}}{b}\right)\right]$

where $\alpha,(0 \leq \alpha<1)$ is called thermal gradient. On using Eqs. (3) and (6), Eqs. (1) and (2), becomes:

$$
\begin{aligned}
T_{s}= & \frac{1}{2} \omega^{2} \rho_{0} l_{0} \int_{0}^{a} \int_{0}^{b}\left[\left(1-m_{1} \Lambda\right)\left(1+\beta \frac{\zeta^{\prime}}{a}\right)\right] \Phi^{2} d \psi^{\prime} d \zeta^{\prime}, \\
V_{s}= & \frac{E_{0} l_{0}^{3}}{24 \cos ^{4} \theta} \int_{0}^{a} \int_{0}^{b}\left[\frac{\left.\left\{1-\alpha\left(1-\frac{\zeta^{\prime 2}}{a^{2}}\right)\left(1-\frac{\psi^{\prime 2}}{b^{2}}\right)\right\}\left(1+\beta \frac{\zeta^{\prime}}{a}\right)^{3}\right]}{\left[1-v_{0}^{2}\left(1-m_{2} \Lambda\right)^{2}\right]}\right] \\
& {\left[\begin{array}{l}
\left(\frac{\partial^{2} \Phi}{\partial \zeta^{\prime 2}}\right)^{2}-4 \Upsilon \sin \theta\left(\frac{\partial^{2} \Phi}{\partial \zeta^{\prime 2}}\right)\left(\frac{\partial^{2} \Phi}{\partial \zeta^{\prime} \partial \psi^{\prime}}\right) \\
+2 \Upsilon^{2}\left[\sin ^{2} \theta+v_{0}\left(1-m_{2} \Lambda\right) \cos ^{2} \theta\right]\left(\frac{\partial^{2} \Phi}{\partial \zeta^{\prime 2}}\right)\left(\frac{\partial^{2} \Phi}{\partial \psi^{\prime 2}}\right) \\
+2 \Upsilon^{2}\left[1+\sin ^{2} \theta-v_{0}\left(1-m_{2} \Lambda\right) \cos ^{2} \theta\right]\left(\frac{\partial^{2} \Phi}{\partial \zeta^{\prime} \partial \psi^{\prime}}\right)^{2} \\
-4 \Upsilon^{3} \sin \theta\left(\frac{\partial^{2} \Phi}{\partial \zeta^{\prime} \partial \psi^{\prime}}\right)\left(\frac{\partial^{2} \Phi}{\partial \psi^{\prime 2}}\right)+\Upsilon^{4}\left(\frac{\partial^{2} \Phi}{\partial \psi^{\prime 2}}\right)^{2}
\end{array}\right] d \psi^{\prime} d \zeta^{\prime}, }
\end{aligned}
$$

where $\Lambda=\left(1-\sqrt{1-\frac{\zeta^{\prime 2}}{a^{2}}}\right)$ and $\Upsilon=\frac{a}{b}$.

It is also considered that parallelogram plate is clamped along all the four edges. Therefore, boundary conditions are: 
$\Phi=\frac{\partial \Phi}{\partial \zeta^{\prime}}=0, \quad \zeta^{\prime}=0, a, \quad \Phi=\frac{\partial \Phi}{\partial \psi^{\prime}}=0, \quad \psi^{\prime}=0, b$

The deflection function which satisfy Eq. (9) is represented by:

$\Phi\left(\zeta^{\prime}, \psi^{\prime}\right)=\left[\begin{array}{c}{\left[\left(\frac{\zeta^{\prime}}{a}\right)^{2}\left(\frac{\psi^{\prime}}{b}\right)^{2}\left(1-\frac{\zeta^{\prime}}{a}\right)^{2}\left(1-\frac{\psi^{\prime}}{b}\right)^{2}\right]} \\ {\left[\Omega_{1}+\Omega_{2}\left(\frac{\zeta^{\prime}}{a}\right)\left(\frac{\psi^{\prime}}{b}\right)\left(1-\frac{\zeta^{\prime}}{a}\right)\left(1-\frac{\psi^{\prime}}{b}\right)\right]}\end{array}\right]$,

where $\Omega_{1}$ and $\Omega_{2}$ are arbitrary constants.

\section{Solution of model for time period}

Rayleigh Ritz technique (i.e., maximum strain energy $V_{s}$ must equal to maximum kinetic energy $T_{s}$ ) is applied to obtain solution of the model. Therefore, we must have:

$\delta\left(V_{s}-T_{s}\right)=0$

Using Eqs. (7) and (8), Eq. (11) becomes:

$\delta\left(V_{s}^{*}-\lambda^{2} T_{s}^{*}\right)=0$,

where:

$$
\begin{gathered}
V_{s}^{*}=\frac{1}{\cos ^{4} \theta} \int_{0}^{a} \int_{0}^{b}\left[\frac{\left\{1-\alpha\left(1-\frac{\zeta^{\prime 2}}{a^{2}}\right)\left(1-\frac{\psi^{\prime 2}}{b^{2}}\right)\right\}\left(1+\beta \frac{\zeta^{\prime}}{a}\right)^{3}}{\left[1-v_{0}^{2}\left(1-m_{2} \Lambda\right)^{2}\right]}\right] \\
\cdot\left[\begin{array}{c}
\left(\frac{\partial^{2} \Phi}{\partial \zeta^{\prime 2}}\right)^{2}-4 \Upsilon \sin \theta\left(\frac{\partial^{2} \Phi}{\partial \zeta^{\prime 2}}\right)\left(\frac{\partial^{2} \Phi}{\partial \zeta^{\prime} \partial \psi^{\prime}}\right) \\
+2 \Upsilon^{2}\left[\sin ^{2} \theta+v_{0}\left(1-m_{2} \Lambda\right) \cos ^{2} \theta\right]\left(\frac{\partial^{2} \Phi}{\partial \zeta^{\prime 2}}\right)\left(\frac{\partial^{2} \Phi}{\partial \psi^{\prime 2}}\right) \\
+2 \Upsilon^{2}\left[1+\sin ^{2} \theta-v_{0}\left(1-m_{2} \Lambda\right) \cos ^{2} \theta\right]\left(\frac{\partial^{2} \Phi}{\partial \zeta^{\prime} \partial \psi^{\prime}}\right)^{2}
\end{array}\right] d \psi^{\prime} d \zeta^{\prime}, \\
-4 \Upsilon^{3} \sin \theta\left(\frac{\partial^{2} \Phi}{\partial \zeta^{\prime} \partial \psi^{\prime}}\right)\left(\frac{\partial^{2} \Phi}{\partial \psi^{\prime 2}}\right)+\Upsilon^{4}\left(\frac{\partial^{2} \Phi}{\partial \psi^{\prime 2}}\right)^{2} \\
T_{s}^{*}=\int_{0}^{a} \int_{0}^{b}\left[\left(1-m_{1} \Lambda\right)\left(1+\beta \frac{\zeta^{\prime}}{a}\right)\right] \Phi^{2} d \psi^{\prime} d \zeta^{\prime} .
\end{gathered}
$$

here $\lambda^{2}=12 \rho_{0} \omega^{2} a^{4} / E_{0} l_{0}^{2}$ is frequency parameter. Eq. (12) consists of two unknown constants $\Omega_{1}$ and $\Omega_{2}$. These two unknowns could be calculated as:

$\frac{\partial}{\partial \Omega_{n}}\left(V_{s}^{*}-\lambda^{2} T_{s}^{*}\right)=0, \quad n=1,2$.

After simplifying Eq. (13) we get:

$r_{n 1} \Omega_{1}+r_{n 2} \Omega_{2}=0, \quad n=1,2$. 
To obtain frequency equation, the determinant of coefficient matrix of Eq. (14) must zero:

$\left|\begin{array}{ll}r_{11} & r_{12} \\ r_{21} & r_{22}\end{array}\right|=0$

where coefficients $r_{11}, r_{12}=r_{21}, r_{22}$ comprises plate parameters and frequency parameter $(\lambda)$.

Time period of vibration is calculated as:

$K=\frac{2 \pi}{\lambda}$

where $\lambda$ is frequency obtained from Eq. (15).

\section{Results and discussion}

Time period (in seconds) for vibrational frequency of non-homogeneous and non-uniform parallelogram plate is calculated on different variation of plate parameters (taper constant $\beta$, thermal gradient $\alpha$, non-homogeneity constants $m_{1}, m_{2}$ and skew angle $\theta$ ) and presented with the help of tables.

Time period for three different values of thermal gradient $\alpha$ and non homogeneity constants $m_{1}, m_{2}$ i.e., $\alpha=m_{1}=m_{2}=0.2,0.4,0.6$ is presented in Table 1 , corresponding to taper constant $\beta$. Table 1 conclude that increasing in taper constant $\beta$ results the decreasing in time period for all the three cases mentioned in Table 1. On the other aspect, increasing in combined value of thermal gradient $\alpha$ and non-homogeneity constants $m_{1}, m_{2}$ results the increasing in time period.

Time period for three different value of taper constant $\beta$ and non homogeneity constants $m_{1}$, $m_{2}$ i.e., $\beta=m_{1}=m_{2}=0.2,0.4,0.6$ tabulated in Table 2 with respect to thermal gradient $\alpha$. Here, increasing in thermal gradient $\alpha$ implies the increasing in time period and increasing in combined value of taper constant $\beta$ and non homogeneity constants $m_{1}, m_{2}$ results the decreasing in time period.

Table 1. Time period $K[s]$ vs taper constant $\beta$ for $\theta=45^{\circ}, a / b=1.5$

\begin{tabular}{|c|c|c|c|c|c|c|}
\hline \multirow{2}{*}{$\beta$} & \multicolumn{2}{|c|}{$\alpha=m_{1}=m_{2}=0.2$} & \multicolumn{2}{c|}{$\alpha=m_{1}=m_{2}=0.4$} & $\alpha=m_{1}=m_{2}=0.6$ \\
\cline { 2 - 7 } & $K_{1}$ & $K_{2}$ & $K_{1}$ & $K_{2}$ & $K_{1}$ & $K_{2}$ \\
\hline 0.0 & 0.013660 & 0.053844 & 0.014189 & 0.055826 & 0.014746 & 0.057922 \\
\hline 0.2 & 0.012400 & 0.048836 & 0.012875 & 0.050595 & 0.013374 & 0.052443 \\
\hline 0.4 & 0.011330 & 0.044576 & 0.011761 & 0.046147 & 0.012212 & 0.047796 \\
\hline 0.6 & 0.010415 & 0.040932 & 0.010809 & 0.042352 & 0.011220 & 0.043835 \\
\hline 0.8 & 0.009628 & 0.037793 & 0.009989 & 0.039085 & 0.010367 & 0.040432 \\
\hline 1.0 & 0.008945 & 0.035073 & 0.009279 & 0.036257 & 0.009627 & 0.037489 \\
\hline
\end{tabular}

Table 2. Time period $K[s]$ vs thermal gradient $\alpha$ for $\theta=45^{\circ}, a / b=1.5$

\begin{tabular}{|c|c|c|c|c|c|c|}
\hline \multirow{2}{*}{$\alpha$} & \multicolumn{2}{|c|}{$\beta=m_{1}=m_{2}=0.2$} & \multicolumn{2}{c|}{$\beta=m_{1}=m_{2}=0.4$} & \multicolumn{2}{c|}{$\beta=m_{1}=m_{2}=0.6$} \\
\cline { 2 - 7 } & $K_{1}$ & $K_{2}$ & $K_{1}$ & $K_{2}$ & $K_{1}$ & $K_{2}$ \\
\hline 0.0 & 0.012103 & 0.047671 & 0.011202 & 0.043976 & 0.010424 & 0.040772 \\
\hline 0.2 & 0.012400 & 0.048836 & 0.011471 & 0.045022 & 0.010670 & 0.041720 \\
\hline 0.4 & 0.012719 & 0.050093 & 0.011761 & 0.046147 & 0.010935 & 0.042738 \\
\hline 0.6 & 0.013065 & 0.051450 & 0.012073 & 0.047363 & 0.011220 & 0.043835 \\
\hline 0.8 & 0.013440 & 0.052993 & 0.012412 & 0.048679 & 0.011529 & 0.045019 \\
\hline
\end{tabular}

Time period for three different value of thermal gradient $\alpha$ and tapering constant $\beta$ i.e., $\alpha=\beta=0.2,0.4,0.6$ is tabulated in Table 3 corresponding to simultaneous variation in non-homogeneity constants $m_{1}, m_{2}$. Here also increasing in non-homogeneity constants $m_{1}, m_{2}$ results the increase in time period for all the three value of thermal gradient $\alpha$ and tapering constant $\beta$ but wih less rate of increment. Also increasing in combined value of thermal gradient 
$\alpha$ and tapering constant $\beta$ results the decrease in the time period.

Time period for three different values of thermal gradient $\alpha$, taper constant $\beta$ and non-homogeneity constants $m_{1}, m_{2}$ i.e., $\alpha=\beta=m_{1}=m_{2}=0.2,0.4,0.6$ is accommodated in Table 4 corresponding to skew angle $\theta$. Increasing in skew angle as well as in the combined value of thermal gradient $\alpha$, taper constant $\beta$ and non-homogeneity constants $m_{1}, m_{2}$, results the decrease in time period. The rate of decrement with increasing value of skew angle $\theta$ is very high in comparison to rate of decrement with the combined increasing value of other plate parameters.

Table 3. Time period $K[s]$ vs non-homogeneity $m_{1}, m_{2}$ for $\theta=45^{\circ}, a / b=1.5$

\begin{tabular}{|c|c|c|c|c|c|c|}
\hline \multirow{2}{*}{$m_{1}, m_{2}$} & \multicolumn{2}{|c|}{$\alpha=\beta=0.2$} & \multicolumn{2}{c|}{$\alpha=\beta=0.4$} & \multicolumn{2}{c|}{$\alpha=\beta=0.6$} \\
\cline { 2 - 7 } & $K_{1}$ & $K_{2}$ & $K_{1}$ & $K_{2}$ & $K_{1}$ & $K_{2}$ \\
\hline 0.0 & 0.012238 & 0.048318 & 0.011463 & 0.045208 & 0.010808 & 0.042559 \\
\hline 0.2 & 0.012400 & 0.048839 & 0.011616 & 0.045691 & 0.010954 & 0.043015 \\
\hline 0.4 & 0.012552 & 0.049329 & 0.011761 & 0.046150 & 0.011091 & 0.043439 \\
\hline 0.6 & 0.012696 & 0.049791 & 0.011897 & 0.046577 & 0.011220 & 0.043835 \\
\hline 0.8 & 0.012832 & 0.050222 & 0.012024 & 0.046970 & 0.011340 & 0.044199 \\
\hline
\end{tabular}

Table 4. Time period $K[s]$ vs skew angle $\theta$ for $a / b=1.5$

\begin{tabular}{|c|c|c|c|c|c|c|}
\hline \multirow{2}{*}{$\theta$} & \multicolumn{2}{|c|}{$\alpha=\beta=m_{1}=m_{2}=0.4$} & \multicolumn{2}{c|}{$\alpha=\beta=m_{1}=m_{2}=0.4$} & $\alpha=\beta=m_{1}=m_{2}=0.6$ \\
\cline { 2 - 7 } & $K_{1}$ & $K_{2}$ & $K_{1}$ & $K_{2}$ & $K_{1}$ & $K_{2}$ \\
\hline 0 & 0.025592 & 0.102547 & 0.024292 & 0.097031 & 0.023189 & 0.092310 \\
\hline 15 & 0.023771 & 0.095011 & 0.022559 & 0.089878 & 0.021533 & 0.085483 \\
\hline 30 & 0.018887 & 0.074999 & 0.017919 & 0.070912 & 0.017101 & 0.067406 \\
\hline 45 & 0.012400 & 0.048836 & 0.011761 & 0.046147 & 0.011220 & 0.043835 \\
\hline 60 & 0.006108 & 0.023881 & 0.005791 & 0.022555 & 0.005524 & 0.021409 \\
\hline
\end{tabular}

\section{Conclusions}

Present model provides time period of parallelogram plate on different variation of plate parameters. From the results discussion, we conclude that increasing in taper constant $\beta$ and skew angle $\theta$, results the decrease in time period as shown in Tables 1 and Table 4. But increasing in thermal gradient $\alpha$ and non-homogeneity constants (simultaneous variation) $m_{1}, m_{2}$, results the increase in time period as shown in Tables 2 and Table 3. The present model provides a good appropriate data for time period of frequency which will be helpful for structural design.

\section{References}

[1] Nair P. S., Durvasula S. Vibration of skew plates. Journal of Sound and Vibration, Vol. 26, 1973, p. 1-19.

[2] Wang X., Wang Y., Yuan Z. Accurate vibration analysis of skew plates by the new version of the differential quadrature method. Applied Mathematical Modelling, Vol. 38, 2014, p. 926-937.

[3] Bacciocchi M., Xeisenberger M., Fantuzzi N., Tornabene F., Viola E. Vibration analysis of variable thickness plates and shells by the Generalized Differential Quadrature method. Composite Structures, Vol. 156, 2016, p. 218-237.

[4] Malekzadeh P., Alibeygi Beni A. Free vibration of functionally graded arbitrary straight-sided quadrilateral plates in thermal environment. Composite Structures, Vol. 92, 2010, p. 2758-2767.

[5] Shenas G., Malekzadeh P. Free vibration of functionally graded quadrilateral microplates in thermal environment. Thin-Walled Structures, Vol. 106, 2016, p. 294-315.

[6] Li Q., Iu V. P., Kou K. P. Three-dimensional vibration analysis of functionally graded material plates in thermal environment. Journal of Sound and Vibration, Vol. 324, 2009, p. 733-750.

[7] Pandey S., Pradyumna S. Free vibration of functionally graded sandwich plates in thermal environment using a Layerwise theory. European Journal of Mechanics - A/Solids, Vol. 51, 2015, p. 55-66.

[8] Zhou L., Zheng W. X. Vibration of skew plates by the MLS-Ritz method. International Journal of Mechanical Sciences, Vol. 50, 2008, p. 1133-1141. 
[9] Ashour A. S. The free vibration of symmetrically angle-ply laminated fully clamped skew plates. Journal of Sound and Vibration, Vol. 323, 2009, p. 444-450.

[10] Dozio L., Carrera E. Ritz analysis of vibrating rectangular and skew multilayered plates based on advanced variable-kinematic models. Composite Structures, Vol. 94, 2012, p. 2118-2128.

[11] Singh B., Saxena V. Transverse vibration of skew plates with variable thickness. Journal of Sound and Vibration, Vol. 206, 1997, p. 1-13.

[12] Thangam Babu P. V., Reddy D. V. Frequency analysis of skew orthotropic plates by the finite strip method. Journal of Sound and Vibration, Vol. 18, 1971, p. 465-474.

[13] Sharma A., Raghav A. K., Kumar V. Mathematical study of vibration on non-homogeneous parallelogram plate with thermal gradient. International Journal of Mathematical Sciences, Vol. 36, 2016, p. 1801-1809.

[14] Sharma A. Vibration of skew plate with circular variation in thickness and Poisson's ratio. Mechanics and Mechanical Engineering, Vol. 22, 2018, p. 43-52.

[15] Sharma A., Sharma A. K., Kumar V. Effect of density and Poisson's ratio on thermal induced vibration of parallelogram plate. Journal of Vibroengineering, Vol. 20, 2018, p. 1288-1298.

[16] Sharma A. Vibrational frequencies of parallelogram plate with circular variations in thickness. Soft Computing: Theories and Applications, Advances in Intelligent Systems and Computing, Springer Nature, Singapore, Vol. 583, 2018, p. 317-326.

[17] Sharma A., Kumar P. Natural vibration of square plate with circular variation in thickness. Soft Computing: Theories and Applications, Advances in Intelligent Systems and Computing, Springer Nature, Singapore, Vol. 742, 2019, p. 311-319.

[18] Kumar A., Lather N., Bhardwaj R., Mani N., Sharma A. Effect of linear variation in density and circular variation in Poisson's ratio on time period of vibration of rectangular plate. Vibroengineering Procedia, Vol. 21, 2019, p. 14-19. 